\title{
First and Second Line Chemotherapeutic Regimens for Non-Small Cell Lung Carcinomas - The Efficacy of Platinum, Non-Platinum and Combination Therapy: A Literature Review
}

\author{
Anita Michael ${ }^{1}$, Alaine Ainsley ${ }^{1}$, Alan Joseph ${ }^{1}$, Nusrat Jahan ${ }^{2,} 3$ \\ 1. Internal Medicine, California Institute of Behavioral Neurosciences \& Psychology, Fairfield, USA 2. Cardiology, Rush \\ Medical Center, Chicago, USA 3. Cardiology, California Institute of Behavioral Neurosciences \& Psychology, Fairfield, \\ USA
}

Corresponding author: Anita Michael, anitamichael13@gmail.com

\begin{abstract}
Non-small cell lung carcinomas (NSCLC) account for a major part of all lung cancer diagnoses. The current literature review is aimed to analyze the varied chemotherapeutic treatment regimens available and to provide a standard for their use in the present and future scenarios. The current literature review focuses on platinum, non-platinum and combination therapeutic modalities, in the first and second line setting. The review also ensures that docetaxel and/or gemcitabine is a part of the study. A PubMed search for NSCLC identified 70,077 articles. A total of 36 research articles were obtained following the application of keywords and inclusion/exclusion criteria to narrow down our search to meet with the research objective. These articles consider NSCLC and chemotherapeutic treatment modalities as its primary endpoint. These 36 articles included 15 randomized clinical trials, five randomized control trials, five retrospective cohort studies, one case-control study, six review articles and four observational studies. Our analysis shows that there is an increasing potential for the use of non-platinum based drugs in the clinical setting with an efficacy that is at par with that of platinum-based treatment modalities. In fact, the studies have proven a greater advantage with the use of combination therapy (non-platinum + platinum), which can be readily applied as an alternative in the clinical setting while the use of non-platinum drugs (other than docetaxel) as a monotherapy or in combination with other non-platinum based drugs does require further research.
\end{abstract}

Received 10/13/2020 Review began 10/27/2020 Review ended 11/11/2020 Published 11/22/2020

() Copyright 2020 Michael et al. This is an open access article distributed under the terms of the Creative Commons Attribution License CC-BY 4.0., which permits unrestricted use, distribution, and reproduction in any medium, provided the original author and source are credited.
Categories: Internal Medicine, Oncology

Keywords: nsclc, chemotherapy, platinum based, docetaxel, gemcitabine, combination therapy

\section{Introduction And Background}

Non-small cell lung carcinomas (NSCLC) account for about $80-85 \%$ of all lung cancer diagnoses [1]. The main sub-types are adenocarcinoma, squamous cell carcinoma and large cell carcinomas. These carcinomas arise from different types of lung cells but are grouped together as NSCLC. This is because they share a similar prognosis and treatment [2]. Most stage one and stage two NSCLC are treated by surgical resection of the tumor followed by chemotherapeutic regimens [3]. However, the initial signs are often ignored and most patients present with advanced-stage NSCLC, which are non-operable at the time of diagnosis. Although the incidence of mortality is high, especially in the case of advanced NSCLC, chemotherapeutic drugs have proved effective in increasing the overall survival (OS) rate [1]. An improvement in the median survival time (MST) by about one and a half to three months can be observed in such individuals [4]. The conventional treatment regimens involve platinum-based chemotherapy. European guidelines recommend a cisplatinbased therapy while American guidelines largely advocate for a platinum-based doublet [5]. Cisplatin is preferred to carboplatin because of better effect on survival as compared to other platinum-based regimens [5]. The last one to two decades saw the advent of non-platinum based regimens involving drugs like docetaxel and gemcitabine into the market which were seen as potential alternatives for the traditional platinum-based therapy. At present, non-platinum based regimens are administered only if platinum-based regimens are contraindicated. Docetaxel is seen as the gold-standard for second-line treatment [5] while gemcitabine is slowly accepted as a first-line drug when given in combination with cisplatin [6]. While platinum-based therapy is associated with an increased risk of toxicity, it is imperative to come up with newer treatment regimens that can replace or reduce this risk of toxicity while proving to be equally efficient in terms of overall survival.

In the presented literature review, the drugs used under the European and American guidelines (carboplatin and cisplatin) will be considered under platinum-based regimens. To narrow down our study, all nonplatinum based regimens will be considered, with inclusion criteria such that docetaxel and gemcitabine are a part of the research study. Docetaxel and gemcitabine are the most commonly used drugs as a part of the non-platinum treatment regimen. Our aim is to compare the efficiency and uses of platinum-based, nonplatinum based, and combination therapy in the treatment of NSCLC. The current literature review hopes to provide greater insight into the available combinations while also giving a standard to compare and 


\section{Cureus}

administer the appropriate first- and second-line treatments for NSCLC.

\section{Review}

\section{Method}

Literature was searched in the PubMed database. Parallel strategies on 'regular keywords' and 'Medical Subject Heading (MeSH) subheadings' were used for data collection. No other database was taken into consideration. Table 1 below further illustrates the above methodology.

\section{Regular Keyword - NSCLC}

Total Records

Regular Keyword NSCLC; Chemotherapy

Total Records

Regular Keyword NSCLC; Chemotherapy Platinum based

Total Records

Regular Keyword NSCLC; Chemotherapy Platinum based docetaxel and gemcitabine

Total Records

MeSH Keyword Carcinoma, non-small cell lung Subheading: Drug Therapy

\section{TABLE 1: Literature Search using Regular and Medical Subject Heading (MeSH) Subheadings} NSCLC: Non-Small Cell Lung Carcinomas

The articles collected from PubMed search results were filtered as per the criteria below:

Inclusion Criteria

Only English language articles with full-text availability were considered. All of these articles were published in Medline journals and were of the following study types: observational studies, cohort studies, case-control studies, review articles, and clinical trials including randomized clinical trials. These studies/review articles were based on first- and second-line chemotherapeutic regimens and involved platinum/non-platinum based therapies like docetaxel and gemcitabine. These studies were based on an adult population (age 19+) as a reference point.

Exclusion Criteria

Non-English language literature and those studies that have not been published in Medline were not considered as a part of the review. All studies conducted in the laboratory on an animal model and the study types involving meta-analysis, case reports and case-series studies have been excluded. Studies/trials conducted on pediatric age groups and on individuals with two or more chemotherapeutic drug therapies were further excluded from the review.

\section{Review}

Table 2 shows the total number of literatures following the application of each inclusion/exclusion criteria to regular and MeSH keywords. 


\section{Cureus}

Regular Keyword - NSCLC

Total Records

70,077

Inclusion/Exclusion

Medline Journal

59,516

English Language

54,317

Humans

53,849

Adults 19+

28,204

Full Text

26,405

Regular Keyword NSCLC; Chemotherapy

Total Records

Inclusion/Exclusion

Medline Journal

English Language

26,495

Humans

26,300

Adults 19+

13,629

Full Text

12,599

Regular Keyword NSCLC; Chemotherapy Platinum based

Total Records

Inclusion/Exclusion

Medline Journal

English Language

2093

Humans

2089

Adults 19+

1299

Full Text

1262

Regular Keyword NSCLC; Chemotherapy Platinum based docetaxel and gemcitabine

Total Records

198

Inclusion/Exclusion

Medline Journal

English Language

170

Humans

Adults 19+

Full Text

97

MeSH Keyword Carcinoma, non-small cell lung Subheading: Drug Therapy

Total Records

20,339

Inclusion/Exclusion

Medline Journal

English Language

18,520

Humans

18,418

Adults 19+

9,576 


\section{Cureus}

TABLE 2: Total number of literatures following application of each inclusion/exclusion criteria.

NSCLC: Non-Small Cell Lung Carcinomas, MeSH: Medical Subject Heading

The selected 12,599 articles described chemotherapeutic drug treatment of NSCLC. A total of 12,502 articles were removed due to lack of specificity towards platinum and non-platinum based treatment regimens. On a refined search, 97 full articles were obtained. All of the 97 articles were reviewed and 61 articles were removed. These 61 articles correspond to: two meta-analysis reports, one duplicate/repeated article, two articles with trials that stopped midway or were not possible to extract data, and 56 articles which failed to comply with our interest towards chemotherapeutic treatment regimens (studies that compared chemotherapy along with radiation therapy, compared toxicities without emphasis over efficacy, genetic polymorphisms related to NSCLC, etc.).

Therefore, 36 articles were reviewed in PubMed and these included: 15 randomized clinical trials, five randomized control trials, five cohort studies (all retrospective cohort studies and no prospective cohort studies), four observational studies, one case control study and six review articles. The maximum number of subjects in a study was 13,472 and the minimum was 27 individuals.

\section{A Brief Analysis of Results}

The articles analyzed the efficacy of platinum, non-platinum or combination therapeutic regimens while also advocating towards specific treatment modalities. Nine studies advocate for combination therapy as an effective treatment regimen for first-line NSCLC [7-15], five studies advocate towards non-platinum based therapy as an effective and safer regimen [8,16-19], one study concludes that non-platinum based therapy is an effective regimen compared to combination therapy in first-line NSCLC [20], two studies conclude that both non-platinum based regimens and combination therapy show an equal efficacy [21,22], three therapies indicate similar efficacy between platinum-based and non-platinum based regimens [23-25], two therapies indicate that the efficacy of non-platinum based therapy is similar to the efficacy of other treatment regimens [26,27], one study states that platinum-based regimens showed a higher efficacy and response rate than non-platinum based regimens [28], 12 studies advocate for non-platinum based regimens as a second line treatment regimen [29-40], and two studies advocate towards combination therapy for second-line treatment [41,42]. Most of the articles lean towards the use of combination therapy for first-line treatment and non-platinum based therapy for second-line treatment. Among non-platinum based therapy, studies mostly advocate for the use of docetaxel or the combination of docetaxel with other non-platinum drug therapies.

The statements used in this article have been cited from the corresponding journals and the references are available. The available articles following inclusion/exclusion criteria have also been subjected to a qualitative review so as to ensure that the relevant disease, the chemotherapeutic treatment regimens, population and the outcome can be studied. A summary of the methods and results is given in Figure 1 below. 


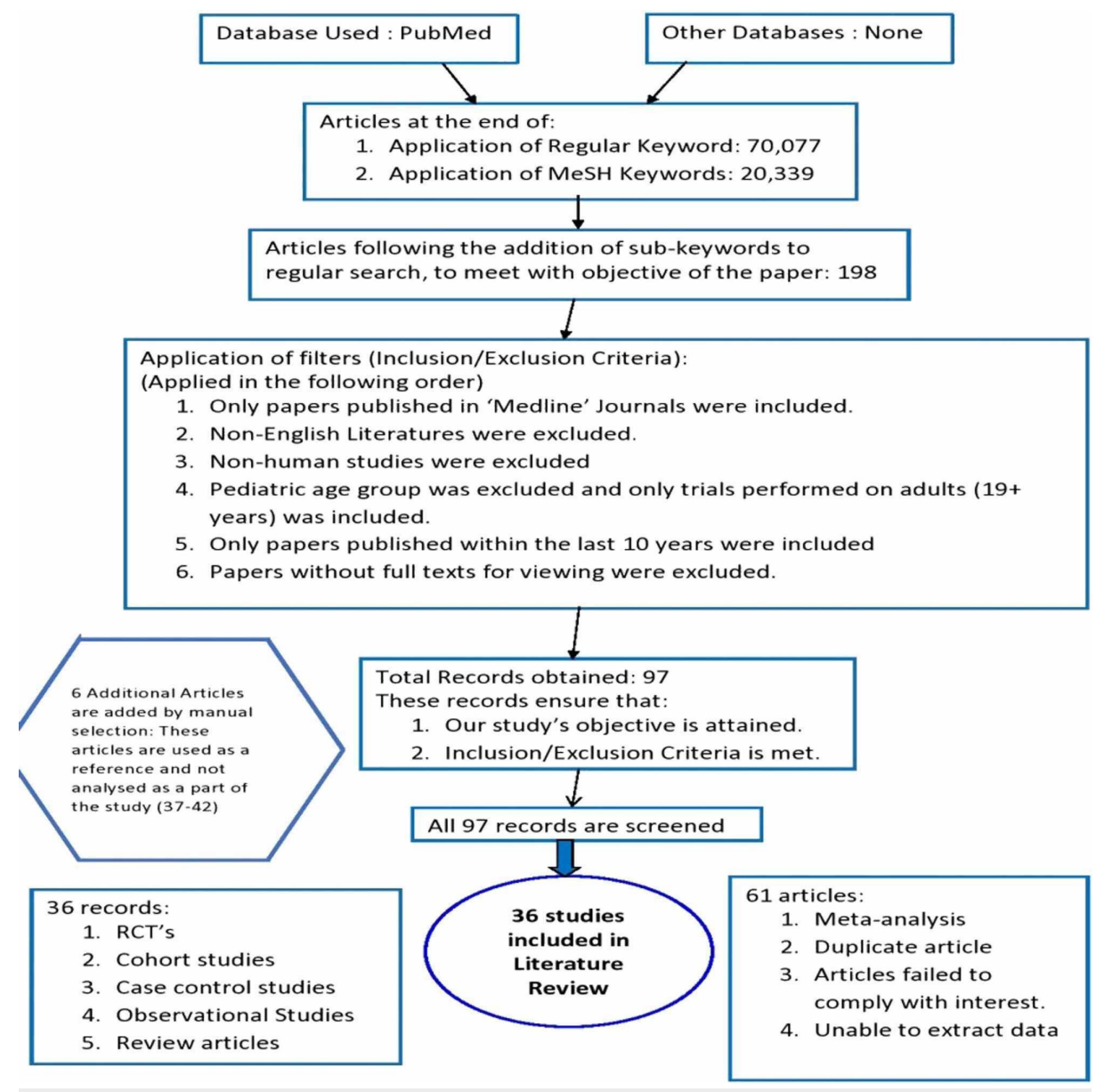

\section{FIGURE 1: Procedure of Literature Review}

RCT: randomized controlled trial

\section{Discussion}

The analysis mainly recognizes the use of non-platinum treatment regimens like docetaxel and gemcitabine or at the least, to incorporate them along with traditional platinum-based modalities of treatment. Platinum-based modalities are associated with an increased risk of toxicity which is imperative to study the use of non-platinum based regimens to eradicate or decrease the toxicities with long term administration. In this article, the efficacy of these regimens as first-line and second-line drugs have been evaluated. A good mean survival time (MST) is seen in both the first- and second-line setting with the use of these regimens. Some of the studies that describe the efficacy of non-platinum-based and combination therapy as first-line treatment regimens are described in Table 3. 


\section{Cureus}

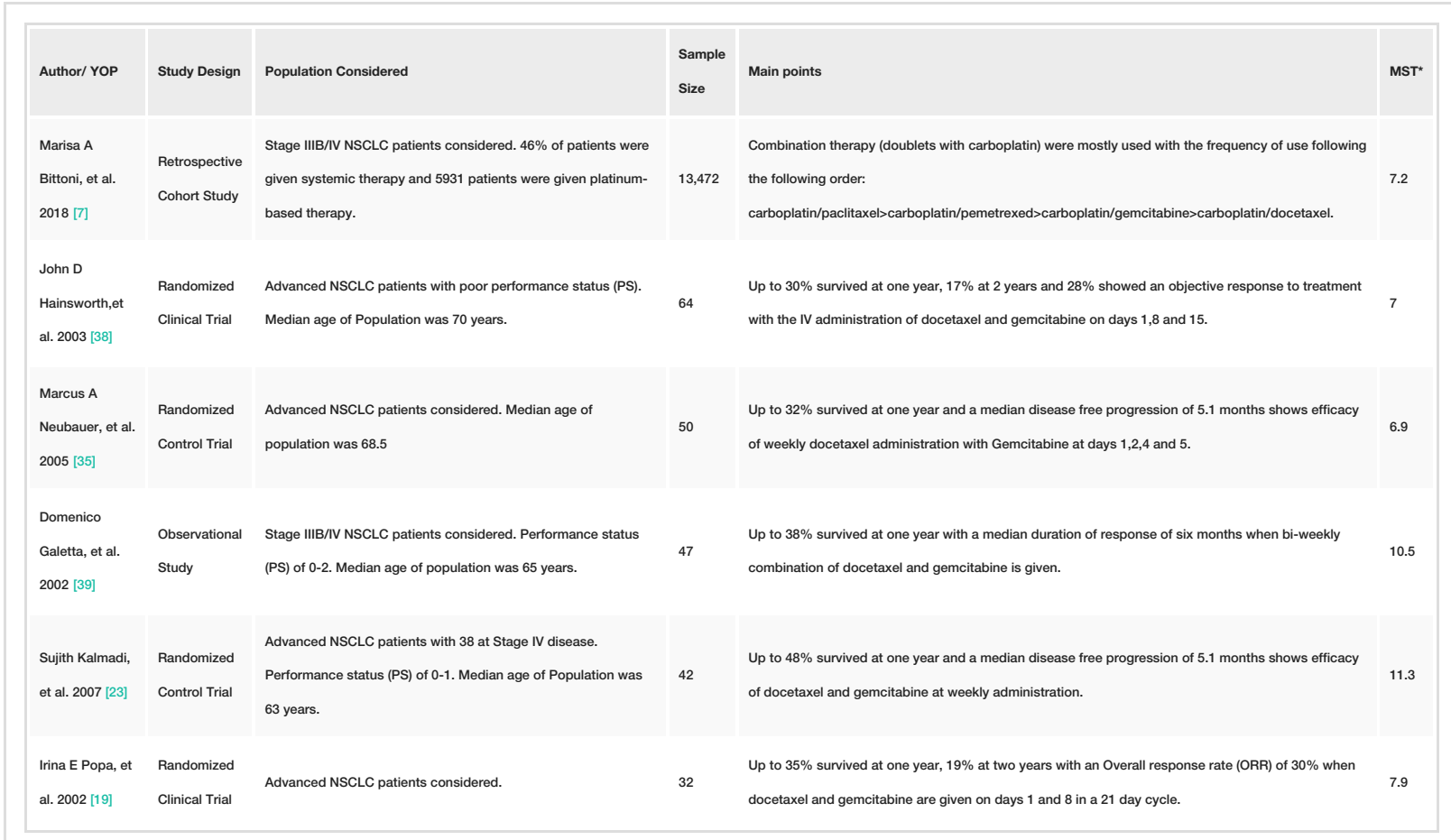

\section{TABLE 3: Summary of studies involving first-line drug regimens in non-small cell lung}

carcinomas (NSCLC).

MST: Median Survival Time; Duration is expressed as months.

ORR: Overall Response Rate; Expressed in Percentage (\%).

Docetaxel and gemcitabine show up to two years survival rates in patients with advanced NSCLC [38] and an MST of up to 11.3 months in the studies evaluated [23]. These statistical evidences provide encouragement towards the use of non-platinum modalities in the treatment of NSCLC, especially advanced NSCLC. Some of the studies that describe the efficacy of non-platinum-based and combination therapy as second-line treatment regimens are described in Table 4. 


\section{Cureus}

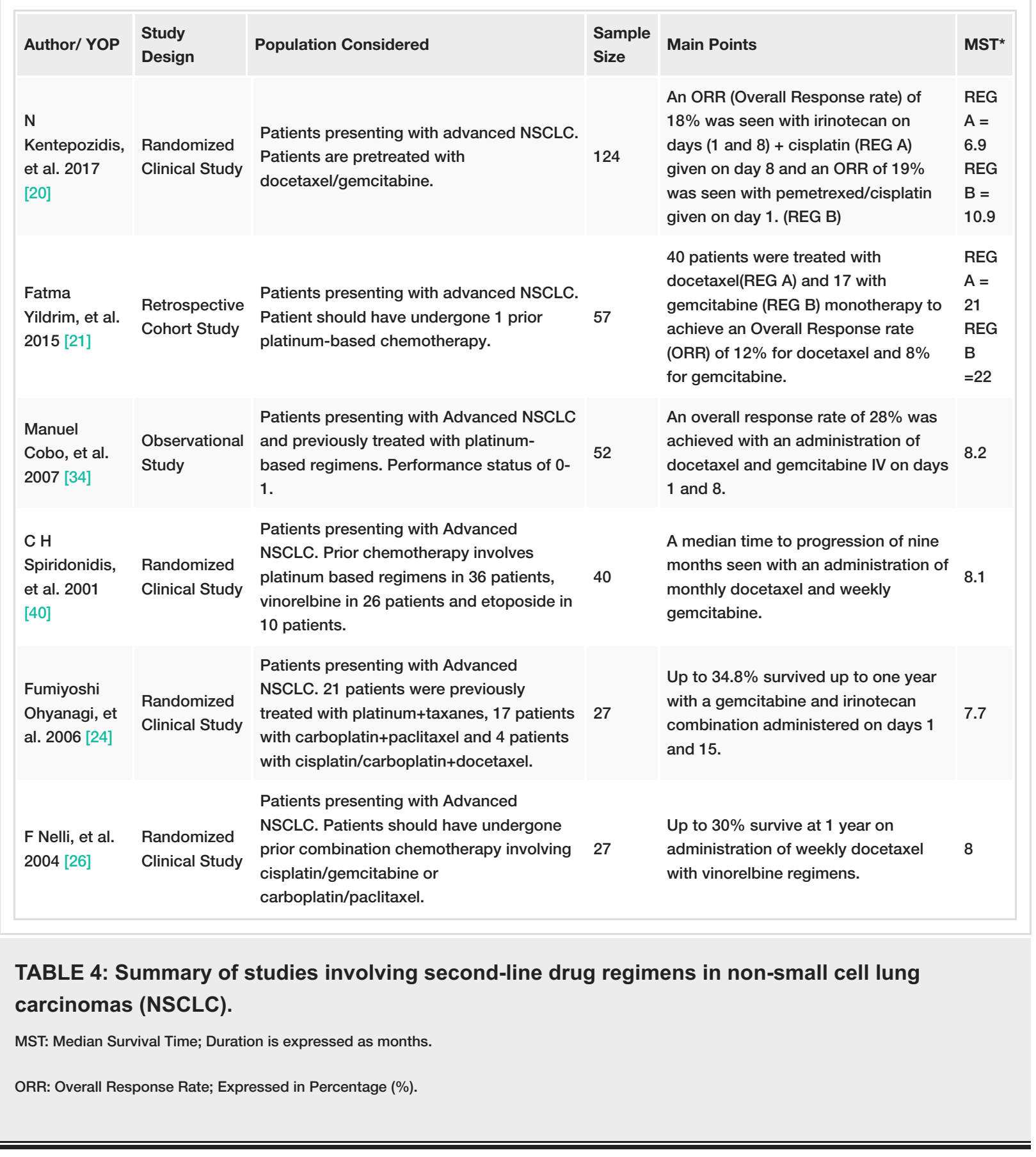

The MST of docetaxel and gemcitabine are 21 and 22 months, respectively, when used as monotherapies [21]. Docetaxel, though considered to be a gold-standard for the second-line treatment of NSCLC, gemcitabine shows equal potential that can be studied further as an alternative to docetaxel.

Overall, in terms of first-line treatment, we found nine articles that advocate for combination therapy and they comprise the following study designs: retrospective cohort study [7], randomized clinical trial [8], randomized control trial [10], case control study [11], randomized clinical trial [12], randomized control trial [13], observational study [14], review article [15]; five articles advocate for non-platinum regimens and they comprise the following study designs: randomized clinical trial [8], review article [16], observational study [17], randomized clinical trial [18], randomized clinical trial [19]; one article (retrospective cohort study [28]) advocates for the use of non-platinum based therapy over combination therapy.

In terms of second-line treatment, 12 articles advocate for the use of non-platinum regimens and they comprise the following study designs: randomized clinical trial [29], randomized clinical trial [30], retrospective cohort study [31], retrospective cohort study [32], randomized clinical trial [33], observational study [34], randomized control trial [35], review article [36], randomized clinical trial [37], randomized clinical trial [38], observational study [39], randomized clinical trial [40] and two studies (randomized clinical trial [41], randomized control trial [42]) advocate for the use of combination therapy. On the basis of the 
articles analyzed, we find an increasing number of articles that advocate towards the use of non-platinum and combination chemotherapeutic modalities.

Limitations of the Review Article

Despite the analysis of efficacy between therapeutic regimens, individual drugs within the non-platinum therapeutic schemes weren’t analyzed separately. The standard drugs, docetaxel and gemcitabine, were used to drive the research forward with little emphasis over the other non-platinum based drugs and their efficacies. An equal number of studies involving different stages of NSCLC and the efficacy of these drugs in each of those stages were impossible to extract with a vast majority of studies leaning towards advanced stage NSCLC's, possibly owing to the lack of diagnosis at the early stages with this disease. Every study was unique in its manner of administration of the same drug, with differences in the dosage/cycles/period of treatment with that specific drug. The accurate dosage or the changes in efficacy and responses with the dosage could not be evaluated in this study. Nevertheless, we have tried to obtain a standard to consider and re-evaluate the uses of newer drug combinations with varied efficacies.

\section{Conclusions}

On the basis of the study results, we can conclude that the current literature review aligns with our research objective to compare the efficiency and the uses of platinum and non-platinum treatment regimens. We can thus conclude that there is an increasing potential for the use of non-platinum based drugs in the clinical setting with an efficacy that is at par with that of platinum based treatment modalities. In fact, the studies have proven a greater advantage with the use of combination therapy (non-platinum + platinum), which can be readily applied as an alternative in the clinical setting. However, as for the use of other non-platinum drugs as a monotherapy or along with other non-platinum drugs, other than that of docetaxel (used in second-line therapy), does require further research over its efficacy and advantages over conventional treatment modalities.

\section{Additional Information \\ Disclosures}

Conflicts of interest: In compliance with the ICMJE uniform disclosure form, all authors declare the following: Payment/services info: All authors have declared that no financial support was received from any organization for the submitted work. Financial relationships: All authors have declared that they have no financial relationships at present or within the previous three years with any organizations that might have an interest in the submitted work. Other relationships: All authors have declared that there are no other relationships or activities that could appear to have influenced the submitted work.

\section{References}

1. Bareschino MA, Schettino C, Rossi A, Maione P, Sacco PC, Zeppa R, Gridelli C: Treatment of advanced non small cell lung cancer. J Thorac Dis. 2011, 3:122-133. 10.3978/j.issn.2072-1439.2010.12.08

2. American Cancer Society: About Lung Cancer . (2019). Accessed: August 3, 2020: https://www.cancer.org/cancer/lung-cancer/about/what-is.html.

3. Lackey A, Donington JS: Surgical management of lung cancer. Semin Intervent Radiol. 2013, 30:133-140. 10.1055/s-0033-1342954

4. Akehurst RL, Beinert T, Crawford J, et al.: Consensus on medical treatment of non-small cell lung cancer . Lung Cancer. 2002, 38:3-7. 10.1016/S0169-5002(02)00259-3

5. Sculier JP: Anticancer treatment for advanced non-small cell lung cancer. Breathe. 2011, 8:124-133. 10.1183/20734735.005011

6. Sculier JP, Moro-Sibilot D: First and second-line therapy for advanced non-small cell lung cancer . Eur Respir J. 2009, 33:915-930. 10.1183/09031936.00132008

7. Bittoni MA, Arunachalam A, Li H, et al.: Real-world treatment patterns, overall survival and occurrence and costs of adverse events associated with first-line therapies for medicare patients 65 years and older with advanced non-small-cell lung cancer: a retrospective study. Clin Lung Cancer. 2018, 19:629-645. 10.1016/j.cllc.2018.04.017

8. Wakelee HA, Dahlberg SE, Keller SM, et al.: Adjuvant chemotherapy with or without bevacizumab in patients with resected non-small-cell lung cancer (E1505): an open-label, multicentre, randomised, phase 3 trial. Lancet Oncol. 2017, 18:1610-1623. 10.1016/S1470-2045(17)30691-5

9. Quoix E, Westeel V, Zalcman G, Milleron B: Chemotherapy in elderly patients with advanced non-small cell lung cancer. Lung Cancer. 2011, 74:364-368. 10.1016/j.lungcan.2011.06.006

10. Takeda K, Negoro S, Tamura T, et al.: Phase III trial of docetaxel plus gemcitabine versus docetaxel in second-line treatment for non-small-cell lung cancer: results of a Japan Clinical Oncology Group trial (JCOG0104). Ann Oncol. 2009, 20:835-841. 10.1093/annonc/mdn705

11. Tada H, Matsui S, Kawahara M, Hosoe S, Hamada C, Fukushima M: Efficacy, toxicity and cost analysis for non-platinum triplet (gemcitabine and vinorelbine, followed by docetaxel) vs. platinum-based chemotherapy in IIIB/IV non-small-cell lung cancer: single-institution experience. Eur J Cancer Care (Engl). 2008, 17:120-126. 10.1111/j.1365-2354.2007.00816.x

12. Kosmas C, Tsavaris N, Syrigos K, et al.: A phase I-II study of bi-weekly gemcitabine and irinotecan as second-line chemotherapy in non-small cell lung cancer after prior taxane + platinum-based regimens. Cancer Chemother Pharmacol. 2007, 59:51-59. 10.1007/s00280-006-0242-5 
13. Pujol J-L, Breton J-L, Gervais R, et al.: Gemcitabine-docetaxel versus cisplatin-vinorelbine in advanced or metastatic non-small-cell lung cancer: a phase III study addressing the case for cisplatin. Ann Oncol. 2005, 16:602-610. 10.1093/annonc/mdi126

14. Syrigos KN, Dannos I, Dionellis G, et al.: Bi-weekly administration of docetaxel and gemcitabine as firstline therapy for non-small cell lung cancer: a phase II study. Anticancer Res. 2005, 25:3489-3493.

15. Rigas JR: Non-platinum based combination chemotherapy: phase I and II trials of docetaxel plus gemcitabine, vinorelbine, or irinotecan. Semin Oncol. 2001, 28:15-20. 10.1016/S0093-7754(01)90220-9

16. Cappuzzo F, Finocchiaro G, Trisolini R, et al.: Perspectives on salvage therapy for non-small-cell lung cancer. Oncology. 2015, 19:989-95.

17. Matsui K, Hirashima T, Nitta T, et al.: A phase I/II study comparing regimen schedules of gemcitabine and docetaxel in Japanese patients with stage IIIB/IV non-small cell lung cancer. Jpn J Clin Oncol. 2005, 35:181187. 10.1093/jico/hyi057

18. Niho S, Kubota K, Goto K, Ohmatsu H, Matsumoto T, Kakinuma R, Nishiwaki Y: Combination second-line chemotherapy with gemcitabine and docetaxel for recurrent non-small-cell lung cancer after platinumcontaining chemotherapy: a phase I/II trial. Cancer Chemother Pharmacol. 2003, 52:19-24. 10.1007/s00280003-0618-8

19. Popa IE, Stewart K, Smith FP, Rizvi NA: A phase II trial of gemcitabine and docetaxel in patients with chemotherapy-naive, advanced non-small cell lung carcinoma. Cancer. 2002, 95:1714-1719. 10.1002/cncr.10843

20. Kentepozidis N, Economopoulou P, Christofyllakis C, et al.: Salvage treatment with irinotecan/cisplatin versus pemetrexed/cisplatin in patients with non-small cell lung cancer pre-treated with a non-platinumbased regimen in the first-line setting: a randomized phase II study of the Hellenic Oncology Research Group (HORG). Clin Transl Oncol. 2017, 19:317-325. 10.1007/s12094-016-1532-y

21. Yıldırım F, Baha A, Yurdakul AS, Ozturk C: Comparison of single agent gemcitabine and docetaxel in second-line therapy for advanced stage non-small cell lung cancer in a university hospital in Turkey. Asian Pac J Cancer Prev. 2015, 16:7859-7863. 10.7314/apjcp.2015.16.17.7859

22. Belani CP: Optimizing chemotherapy for advanced non-small cell lung cancer: focus on docetaxel . Lung Cancer. 2005, 50:3-8. 10.1016/S0169-5002(05)81567-3

23. Kalmadi S, McNeill G, Davis M, Peereboom D, Adelstein D, Mekhail T: Phase II trial of weekly docetaxel and gemcitabine as first-line therapy for patients with advanced non-small cell lung cancer. Med Oncol. 2006, 23:507-513. 10.1385/MO:23:4:507

24. Ohyanagi F, Taguchi F, Horai T, et al.: Phase II study of combination chemotherapy with gemcitabine and irinotecan in patients with advanced non-small-cell lung cancer previously treated with platinumcontaining chemotherapy regimens. Jpn J Clin Oncol. 2006, 36:547-551. 10.1093/jjco/hyl062

25. Georgoulias V, Papadakis E, Alexopoulos A, et al.: Platinum-based and non-platinum-based chemotherapy in advanced non-small-cell lung cancer: a randomised multicentre trial. Lancet. 2001, 357:1478-1484. 10.1016/S0140-6736(00)04644-4

26. Nelli F, Naso G, Ceratti ADP, et al.: Weekly vinorelbine and docetaxel as second-line chemotherapy for pretreated non-small cell lung cancer patients: a phase I-II trial. J Chemother. 2004, 16:392-399. 10.1179/joc.2004.16.4.392

27. Kosmas C, Tsavaris N, Kalofonos HP: Salvage chemotherapy with the gemcitabine/docetaxel combination in non-small cell lung cancer: an overview of recent phase II studies. Med Sci Monit. 2002, 8:58-63.

28. Odabas H, Ulas A, Aydin K, et al.: Is second-line systemic chemotherapy beneficial in patients with nonsmall cell lung cancer (NSCLC): a multicenter data evaluation by the Anatolian Society of Medical Oncology. Tumour Biol. 2015, 36:9641-9648. 10.1007/s13277-015-3728-0

29. Kotsakis A, Kentepozidis N, Emmanouilidis C, et al.: Sequential administration of vinorelbine plus cisplatin and bevacizumab followed by docetaxel plus gemcitabine and bevacizumab compared to docetaxel plus cisplatin and bevacizumab regimen as first-line therapy for advanced or metastatic non-squamous nonsmall cell lung cancer: a multicenter randomized phase II trial of the Hellenic Oncology Research Group (HORG). Lung Cancer. 2015, 88:57-62. 10.1016/j.lungcan.2015.01.012

30. Berghmans T, Lafitte JJ, Scherpereel A, et al.: An ELCWP phase III trial comparing ifosfamide and cisplatin regimens in advanced NSCLC. Anticancer Res. 2013, 33:5477-5482.

31. Inal A, Kaplan MA, Kucukoner M, Urakci Z, Karakus A, Isikdogan A: Cisplatin-based therapy for the treatment of elderly patients with non-small-cell lung cancer: a retrospective analysis of a single institution. Asian Pac J Cancer Prev. 2012, 13:1837-1840. 10.7314/apjcp.2012.13.5.1837

32. Kotsakis A, Hatzidaki D, Vamvakas L, et al.: A retrospective analysis of non-platinum-based first- and second-line chemotherapy in patients with advanced non-small cell lung cancer. Anticancer Res. 2010, 30:4335-4342.

33. Huang CH, Millenson MM, Sherman EJ, et al.: Promising survival in patients with recurrent non-small cell lung cancer treated with docetaxel and gemcitabine in combination as second-line therapy. J Thorac Oncol. 2008, 3:1032-1038. 10.1097/TTO.0b013e31818307c2

34. Cobo M, Gutiérrez V, Alcaide J, et al.: A phase II study of days 1 and 8 combination of docetaxel plus gemcitabine for the second-line treatment of patients with advanced non-small-cell lung cancer and good performance status. Lung Cancer. 2007, 56:255-262. 10.1016/j.lungcan.2006.12.013

35. Neubauer MA, Garfield DH, Kuerfler PR, et al.: Results of a phase II multicenter trial of weekly docetaxel and gemcitabine as first-line therapy for patients with advanced non-small cell lung cancer. Lung Cancer. 2005, 47:121-127. 10.1016/j.lungcan.2004.05.015

36. Ramalingam S: First-line chemotherapy for advanced-stage non-small-cell lung cancer: focus on docetaxel . Clin Lung Cancer. 2005, 7:77-82. 10.3816/CLC.2005.s.014

37. Tas F, Demir C, Camlica H, Ustuner Z, Topuz E: Second-Line docetaxel and gemcitabine combination chemotherapy in patients with non-small-cell lung cancer previously treated with platinum-based chemotherapy: a phase II trial. Med Oncol. 2004, 21:233-240. 10.1385/MO:21:3:233

38. Hainsworth JD, Erland JB, Barton JH, et al.: Combination treatment with weekly docetaxel and gemcitabine for advanced non-small-cell lung cancer in elderly patients and patients with poor performance status: 


\section{Cureus}

results of a Minnie Pearl Cancer Research Network phase II trial. Clin Lung Cancer. 2003, 5:33-38.

10.3816/clc.2003.n.019

39. Galetta D, Gebbia V, Giotta F, et al.: Gemcitabine and docetaxel every 2 weeks in advanced non-small cell lung cancer: a phase II study of the Gruppo Oncologico Italia Meridionale. Lung Cancer. 2002, 38:79-84. 10.1016/s0169-5002(02)00174-5

40. Spiridonidis CH, Laufman LR, Carman L, et al.: Second-line chemotherapy for non-small-cell lung cancer with monthly docetaxel and weekly gemcitabine: a phase II trial. Ann Oncol. 2001, 12:89-94. 10.1023/a:1008306616994

41. Pallis AG, Syrigos K, Kotsakis A, et al.: Second-line paclitaxel/carboplatin versus vinorelbine/carboplatin in patients who have advanced non-small-cell lung cancer pretreated with non-platinum-based chemotherapy: a multicenter randomized phase II study. Clin Lung Cancer. 2011, 12:100-105. 10.1016/i.cllc.2011.03.004

42. William WN, Khuri FR, Fossella FV, et al.: Phase II study of vinorelbine and docetaxel in the treatment of advanced non-small-cell lung cancer as frontline and second-line therapy. Am J Clin Oncol. 2010, 33:148152. 10.1097/COC.0b013e318199fb99 